\title{
Mania symptom profile changes with age
}

Published online: 26 June 2012

(C) Springer Healthcare 2012

MedWire News: A review of published studies shows that there are significant age-group differences in the prominence of specific symptoms of mania among patients with bipolar disorder.

Indeed, the researchers found that anger dyscontrol was the most prominent mania symptom in young people with the mood disorder, whereas disordered thought content was the most prominent mania symptom in adults.

Daniel Safer (Johns Hopkins University School of Medicine, Baltimore, Maryland, USA) and colleagues searched the literature for double-blind, placebo-controlled studies of bipolar disorder patients that included data on Young Mania Rating Scale (YMRS) scores by age group.

In total, 12 studies were included in the final analysis, of which four were conducted in patients with bipolar I disorder only, and eight in those with bipolar I disorder, bipolar II disorder, and bipolar disorder not otherwise specified (NOS).

Examination of the pooled data revealed that, compared with adults, adolescents (aged 10-17 years) with bipolar I disorder had significantly higher YMRS item scores - relative to the total score - for aggression and irritability, at $15.0 \%$ versus $8.7 \%$, and $16.7 \%$ versus $13.9 \%$, respectively.

By contrast, YMRS grandiosity and sexual interest item scores were higher in adults than adolescents, at $15.8 \%$ versus $10.3 \%$, and $5.3 \%$ versus $3.5 \%$, respectively, relative to the total score.

These age-group differences remained similar in analyses of patients with bipolar II disorder, and bipolar disorder NOS, the researchers note.

Safer and team conclude in Comprehensive Psychiatry: "Age-grouped differences in the assessment of bipolar manic disorder need to be taken into account to better understand the psychopathology of mania and to build consistent diagnostic criteria for community care."

By Mark Cowen

Reference

Compr Psychiatry 2012; Advance online publication 